\title{
Revealing the Small-Bundle Internal Structure of Vertically Aligned Single-Walled Carbon Nanotube Films ${ }^{\dagger}$
}

\author{
Erik Einarsson, ${ }^{\ddagger}$ Hidetsugu Shiozawa, ${ }^{\S}$ Christian Kramberger, ${ }^{\S}$ Mark H. Riummeli, ${ }^{\S}$ \\ Alexander Griineis, ${ }^{\S}$ Thomas Pichler, ${ }^{\S}$ and Shigeo Maruyama*,* \\ Department of Mechanical Engineering, The University of Tokyo, Tokyo 113-8656, Japan, and Institute for \\ Solid State and Materials Research (IFW) Dresden, D-01171 Dresden, Germany
}

Received: February 16, 2007; In Final Form: July 29, 2007

\begin{abstract}
Freestanding arrays of vertically aligned single-walled carbon nanotubes (VA-SWNTs) synthesized from alcohol were observed directly by transmission electron microscopy (TEM). These observations revealed that the films are comprised primarily of small bundles, containing 10 or fewer SWNTs per bundle. The absence of large, well-formed bundles is supported by electron diffraction spectra in which no bundle peak is observed. Furthermore, electron energy-loss spectroscopy shows an unusually low $\pi$ plasmon energy, meaning dielectric screening from tube-tube interactions is insignificant. Not only does this suggest a prevalence of small bundles but it also indicates that the optical properties of the VA-SWNT array are determined by the one-dimensional nature of the component SWNTs, rather than the bulk dimensions of the entire SWNT film.
\end{abstract}

\section{Introduction}

Many potential applications of single-walled carbon nanotubes (SWNTs) have been proposed, most of which are based on exploiting the novel physical properties of SWNTs arising from their quasi-one-dimensional structure. ${ }^{1,2}$ This one-dimensionality induces strong anisotropy in many SWNT properties, such as optical absorption; ${ }^{3-5}$ thus, controlling the orientation of SWNTs is highly desirable. After vertically aligned (VA-) synthesis of SWNTs was realized ${ }^{6}$ by the alcohol catalytic chemical vapor deposition (ACCVD) method, ${ }^{7}$ several other groups have reported VA-SWNT synthesis using various techniques, such as water-assisted $\mathrm{CVD},{ }^{8,9}$ oxygen-assisted $\mathrm{CVD},{ }^{10}$ point-arc microwave plasma CVD,${ }^{11}$ molecular-beam CVD, ${ }^{12}$ and hotfilament CVD. ${ }^{13}$ It has also been shown that the catalyst can be optimized to obtain VA-SWNT growth using traditional CVD methods. ${ }^{14,15}$

In addition to controlling the orientation of SWNTs, there is another challenge regarding SWNT synthesis. Many of the unique 1D properties of SWNTs are lost because of bundling of the nanotubes, which not only makes many proposed applications impractical or impossible but also makes measurements of some basic physical properties of SWNTs more challenging. In particular, the effects of bundling on the electronic properties of SWNTs have been addressed in many reports in the literature.

In this paper, we report the first transmission electron microscope (TEM) observations of freestanding VA-SWNTs. These observations show that VA-SWNTs are composed of very small SWNT bundles, a fact that had not been revealed in previous studies on these aligned SWNT arrays. This finding is supported by electron diffraction and electron energy-loss spectroscopy (EELS), the latter also indicating that the electronic

\footnotetext{
† Part of the special issue "Richard E. Smalley Memorial Issue".

* Corresponding author. E-mail: maruyama@photon.t.u-tokyo.ac.jp. Tel/ Fax: +81-3-5800-6983.

$\doteqdot$ The University of Tokyo.

$\S$ Institute for Solid State and Materials Research (IFW) Dresden.
}

properties of the VA-SWNT films are strongly affected by the small bundle size.

\section{Experimental Methods}

2.1. Preparation of Vertically Aligned SWNTs by the ACCVD Method. The VA-SWNTs used in this study were synthesized from ethanol by the ACCVD method. ${ }^{7}$ Details of the synthesis procedure have been described in previous reports, ${ }^{6,16}$ but the general process is as follows. The catalyst is prepared by dip-coating ${ }^{17}$ optically polished quartz glass into a solution of $\mathrm{Co}$ and Mo acetates dissolved in ethanol (metal content $0.01 \mathrm{wt} \%$ each). The substrate is then baked in air at $400{ }^{\circ} \mathrm{C}$ for $5 \mathrm{~min}$, which oxidizes the deposited metals and prevents agglomeration at higher temperatures. Immediately prior to CVD, the sample is heated to the growth temperature (typically $\left.800{ }^{\circ} \mathrm{C}\right)$ under $40 \mathrm{kPa}$ of flowing $\mathrm{Ar} / \mathrm{H}_{2}\left(3 \% \mathrm{H}_{2}, \mathrm{Ar}\right.$ balance, flow rate of $300 \mathrm{sccm})$. After reaching the growth temperature, the $\mathrm{Ar} / \mathrm{H}_{2}$ flow is stopped and ethanol is introduced into the chamber for $10-15 \mathrm{~min}$ at a pressure of $0.8 \mathrm{kPa}$. An SEM image of VA-SWNTs produced by this method is shown in Figure 1a. Figure $1 \mathrm{~b}$ is a corresponding resonance Raman spectrum, which reveals the presence of high-purity SWNTs. ${ }^{18}$ The dominant RBM peak at $180 \mathrm{~cm}^{-1}$ (see inset) has been shown to be indicative of vertical alignment. ${ }^{19}$ During synthesis, the VA-SWNT film thickness was monitored using an in situ optical absorbance technique, ${ }^{16}$ which allows real-time observation of the film thickness up to $\sim 30 \mu \mathrm{m}$. To ensure electron transparency, thin VA-SWNT films were desired, so this in situ monitoring was used to stop the growth of two different VASWNT films at 2 and $7 \mu \mathrm{m}$.

2.2. Transfer onto TEM Grid. After synthesis, the VASWNT film was removed from the quartz substrate on which it was grown and transferred onto a TEM grid using the hotwater-assisted process. ${ }^{20}$ Because this method preserves vertical alignment, the resulting sample was a freestanding VA-SWNT film sitting atop a TEM grid. This is shown by an optical microscope image in Figure $2 \mathrm{a}$ and an illustration of the setup in Figure 2b. A scanning electron microscope (SEM) image 

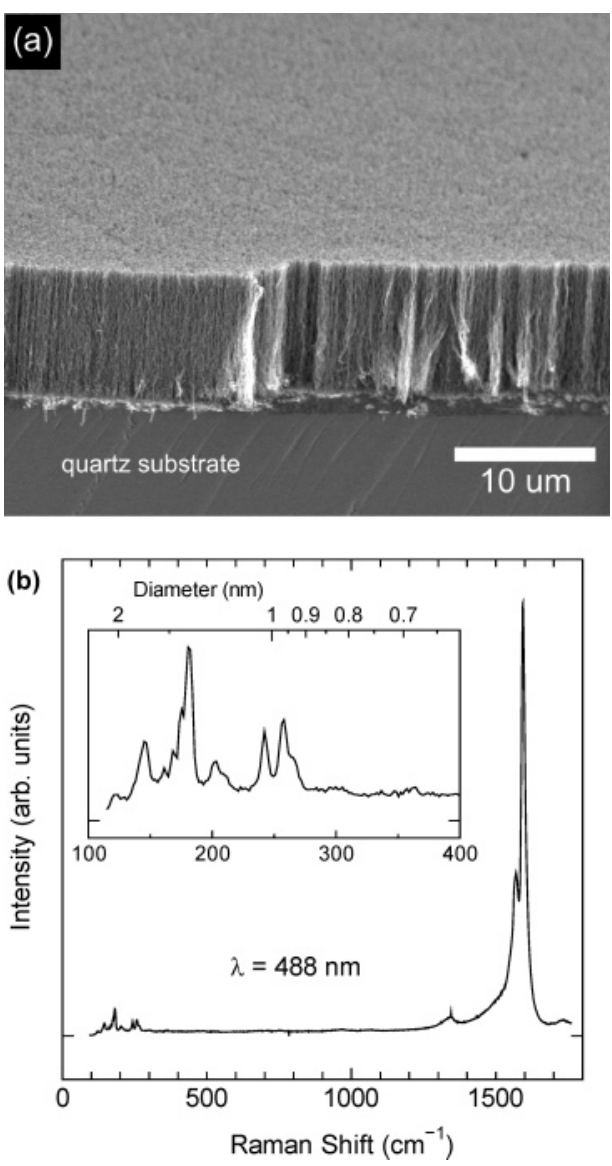

Figure 1. SEM image of VA-SWNTs synthesized from alcohol. The film thickness can be controlled from 1 to $30 \mu \mathrm{m}$. (b) A Raman spectrum representative of VA-SWNTs produced by the alcohol CVD method. RBM peaks are shown in the inset, where the upper scale indicates the SWNT diameters determined from the RBM frequency.

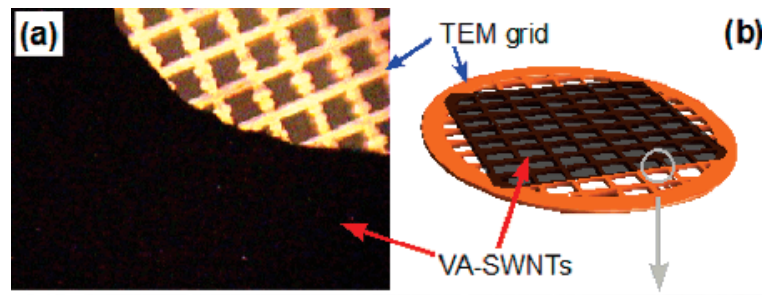

(b)

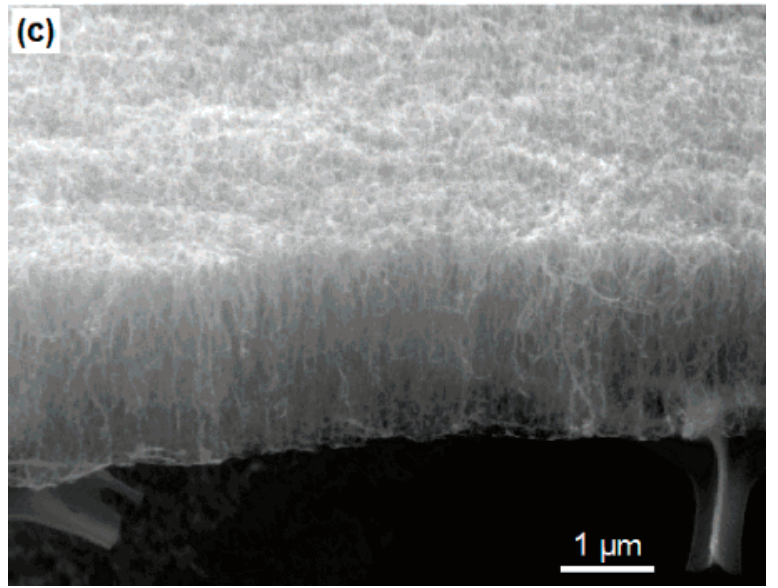

Figure 2. (a) Optical image of VA-SWNT film (black area) sitting atop a TEM grid, (b) illustration of the sample, and (c) SEM image of the film edge after transfer.

(Figure 2c) shows the aligned film after transfer onto the TEM grid. EELS and electron diffraction measurements were per-
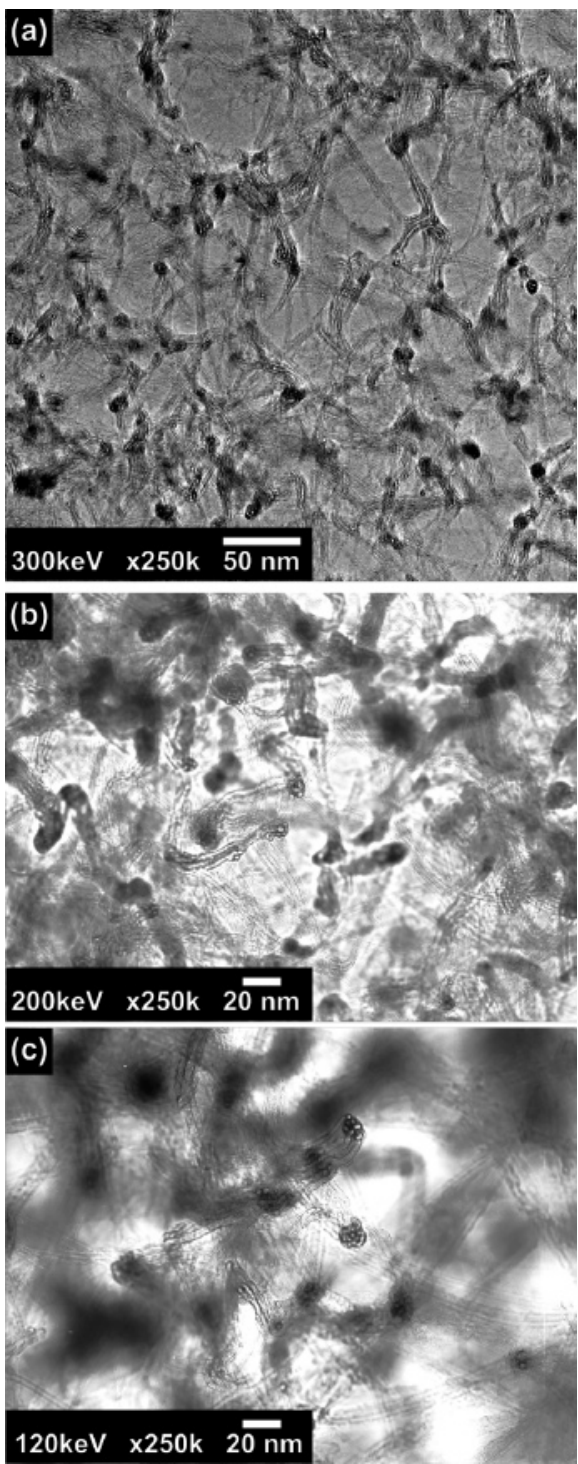

Figure 3. TEM images along the alignment direction of freestanding VA-SWNTs. The cross sections of many small SWNT bundles can be seen. The image in a was taken at $300 \mathrm{keV}$ with a Tecnai F30, and b and c were taken at 200 and $120 \mathrm{kV}$, respectively, using a JEOL JEM2000EX.

formed at room temperature under ultrahigh vacuum using a purpose-built high-resolution spectrometer having good energy and momentum resolution. ${ }^{21}$

\section{Results}

3.1. TEM Observation. The VA-SWNTs synthesized as described in the previous section were observed in a TEM such that the perspective was along the alignment direction, that is, down from the top of the film shown in Figure $2 b$. The image in Figure 3a was taken with an accelerating voltage of $300 \mathrm{kV}$ using an FEI Tecnai F-30 microscope. Despite the thickness of the sample $(2 \mu \mathrm{m})$, its low density $\left(0.035-0.05 \mathrm{~g} \mathrm{~cm}^{-3}\right)$ makes it sufficiently transparent to the electron beam to be clearly observed. Additionally, the high acceleration voltage $(300 \mathrm{kV})$ results in significant transmission through the sample; thus, SWNTs in the foreground and background are not apparent in the image. Increasing magnification reveals that the dark spots dotting the image in Figure $3 \mathrm{a}$ are not metal particles or other impurities but cross-sections of small SWNT bundles (not shown, but similar to Figure $3 \mathrm{~b}$ and $\mathrm{c}$ ). The large number of 


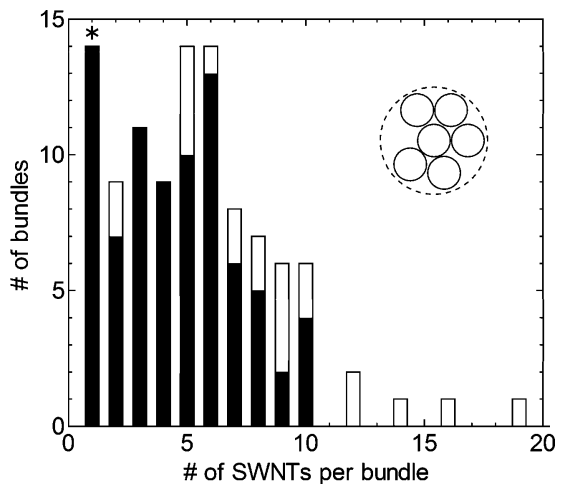

Figure 4. Distribution of bundle sizes for the VA-SWNT array shown in Figure 3a (data obtained using additional images). Filled bars are from clearly observed SWNT bundles, and unfilled bars are estimates based on the size of the bundle when the number of SWNTs in the bundle was not clear from the image. The median size of six SWNTs is shown as an inset. The number of isolated SWNTs (marked by a star) could be much higher than that shown here.

cross sections visible in each image is due to the overall alignment of the SWNTs. Bundle diameters are typically a few nanometers, with each bundle containing roughly 10 or fewer SWNTs. A few isolated SWNTs were also found dispersed throughout the film.

The images shown in Figure $3 \mathrm{~b}$ and $\mathrm{c}$ were taken using reduced acceleration voltages (200 and $120 \mathrm{kV}$, respectively). These images were also obtained using a thicker VA-SWNT film (approximately $7 \mu \mathrm{m}$ ). The lower acceleration voltage and thicker sample made the thickness of the film much more apparent, particularly in Figure 3c, but the internal structure observed in both cases is basically the same.

From the TEM images, however, the SWNTs seem less-well aligned than that indicated by typical SEM observation. It is possible that the exposed SWNTs at a free surface of the VASWNT film differ from the inside.

Several TEM images of the $2-\mu \mathrm{m}$-thick VA-SWNT film (see Figure 3a) were analyzed to determine the frequency of different SWNT bundle sizes. The resulting histogram is shown in Figure 4. The median bundle size was found to be 6 SWNTs, with very few bundles containing more than 10 SWNTs. For these VA-SWNTs, which have an average diameter of $2 \mathrm{~nm}$, the bundle diameter is nearly the same as the number of SWNTs contained in the bundle. This is illustrated by the inset, where the median of six SWNTs is shown. It should be noted, however, that nonideal packing of SWNTs in small bundles was often observed, for example, three SWNTs forming a line, rather than a triangular shape. A rough calculation of the number density of SWNTs based on these observations yielded $2.3 \times 10^{15} \mathrm{~m}^{-2}$, which is in relatively good agreement with the value of $7-10$ $\times 10^{15} \mathrm{~m}^{-2}$ calculated from the mass density of the VA-SWNT film $\left(0.035 \sim 0.05 \mathrm{~g} \mathrm{~cm}^{-3}\right)$ and assuming a SWNT diameter of $2 \mathrm{~nm}$. Because SWNTs not passing through the image plane cannot be counted accurately, a somewhat lower value is expected from the statistical analysis obtained from the TEM images. Furthermore, the presence of isolated SWNTs in these images can be ambiguous; thus, the relative number of isolated SWNTs shown in Figure 4 (marked by a star) is not a reliable value and could be significantly higher.

3.2. Electron Spectroscopy. Electron diffraction spectra obtained from SWNT samples have three characteristic peaks, including the (110) and (100) peaks from the graphitic structure and a large peak at low momentum transfer (near $0.7 \AA^{-1}$ ), which is caused by intertube scattering within SWNT bundles. ${ }^{22}$ Because of the alignment of the VA-SWNTs, we expect an

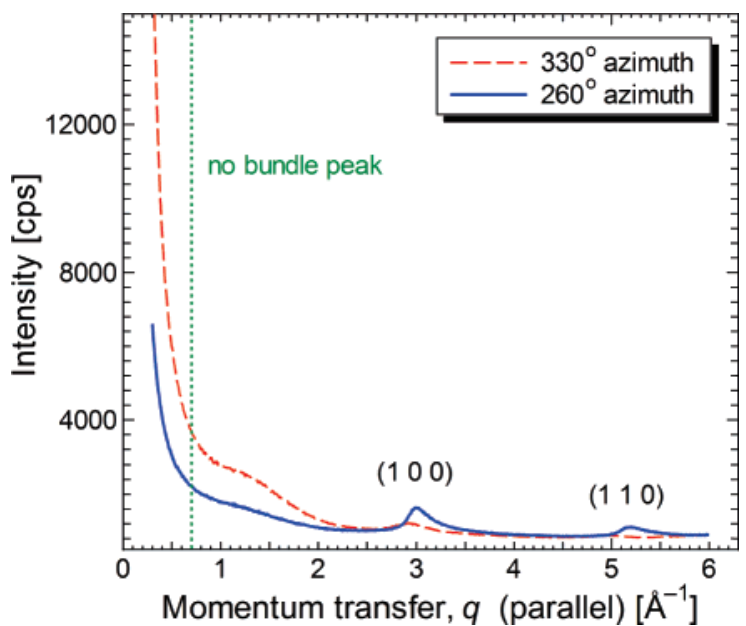

Figure 5. Electron diffraction intensity profiles of the VA-SWNT film showing alignment-induced anisotropy but no bundle peak in the low- $q$ region (expected at dotted line).

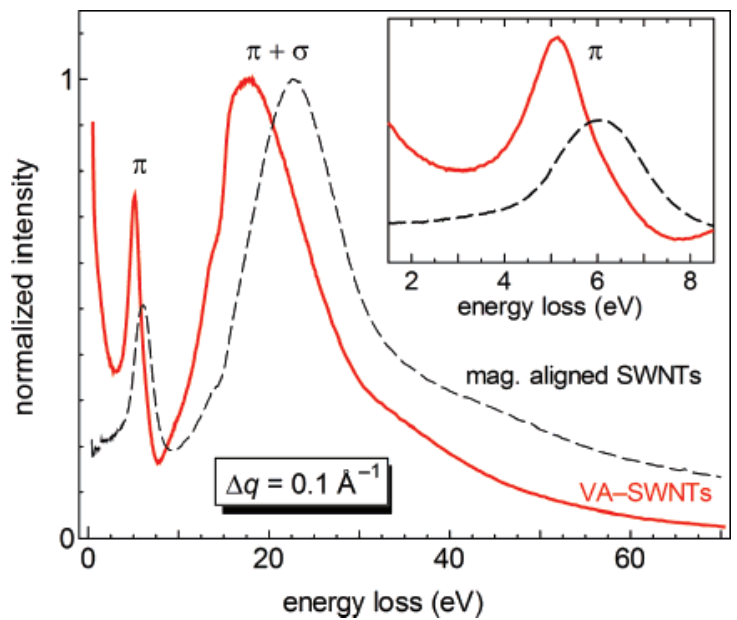

Figure 6. EELS spectra for momentum transfer $\Delta q=0.1 \AA^{-1}$ showing the $\pi$ and $\pi+\sigma$ plasmon peaks for magnetically aligned SWNT bundles (dashed black line, from ref 22) and VA-SWNTs (solid red line). The lower $\pi$ plasmon energy of the VA-SWNTs (see inset) indicates reduced tube-tube interactions.

orientation-dependent anisotropy in the electron scattering spectra, shown in Figure 5. As expected, the (100) and (110) reflections (found at 3 and $5 \AA^{-1}$, respectively) are stronger for incidence along the orientation axis (solid blue line in Figure 5); however, there is no indication of the bundle peak expected at low momentum transfer. The lack of this bundle peak does not imply that SWNT bundles are not present, but it does indicate that the relative population of well-formed SWNT bundles within the film is small. The broad hump between 1 and $2 \AA^{-1}$ is believed to be due to the broad diameter distribution of the SWNTs present in the sample. ${ }^{6}$

High-resolution electron energy-loss spectroscopy (EELS) measurements also indicate an absence of large SWNT bundles within the aligned films. The TEM observations shown above indicate that the bundles comprising the VA-SWNT film typically contain approximately six SWNTs. For bundles of this size, essentially all of the SWNTs are on the "surface" of the bundle, and essentially none are in the interior, or bulk, of the bundle. Hence, one expects very little change in the dielectric response function because of shielding by the surrounding SWNTs.

Figure 6 shows two EELS spectra for low momentum transfer, $\Delta q=0.1 \AA^{-1}$. The solid red line corresponds to the 
VA-SWNTs discussed in this report, and the dashed black line corresponds to magnetically aligned bundles of SWNTs investigated in a previous study. ${ }^{22}$ Analysis of the EELS spectra for these two samples can be found in ref 23 , but here we focus only on the low momentum transfer region $\left(\Delta q=0.1 \AA^{-1}\right)$, which is analogous to optical absorption spectroscopy.

The peaks in the spectra shown in Figure 6 correspond to plasma oscillations (plasmons) ${ }^{24-26}$ in the $\pi$ electron system (for energies between 5 and $7 \mathrm{eV}$ ) and the combined oscillation of the $\pi$ and $\sigma$ electrons (around $20 \mathrm{eV}$ ). The $\pi$ plasmon energy is particularly sensitive to the local dielectric response function, which changes with polarization and the dielectric environment. We see that both the $\pi$ and $\pi+\sigma$ plasmon excitations are found at lower energies in the VA-SWNTs than the magnetically aligned SWNT bundles. ${ }^{22}$ The $\pi$ plasmon peak (Figure 6 inset) appears at $5 \mathrm{eV}$, which is a full $1 \mathrm{eV}$ lower than for the magnetically aligned SWNT sample, and quite close to the value of $4.5 \mathrm{eV}$ obtained previously from polarized optical absorption measurements of VA-SWNTs. ${ }^{4}$

Considering the electron diffraction results and the sensitivity of the $\pi$ plasmon energy to local shielding effects, this reduced plasmon energy is very likely due to the small SWNT bundles within the VA-SWNT film. A detailed analysis of the EELS spectra will be addressed in a separate report, but these initial results indicate that the aligned SWNTs consist of sufficiently small bundles that dielectric screening is minimal; thus, the 1D optical nature of the component SWNTs is retained. Early calculations by Lin et al. ${ }^{27}$ show the $\pi$ plasmon energy for individual SWNTs should be lower than for bundles, in agreement with the measurements presented here.

\section{Conclusions}

We report the first TEM observations of a vertically aligned (VA-)SWNT film along the alignment direction. These observations reveal that the VA-SWNTs form unexpectedly small bundles, typically containing 10 or fewer SWNTs. Electron diffraction and electron energy-loss spectra obtained from these VA-SWNTs show little indication of bundling, because of the lack of a bundle peak in the diffraction spectra, and a significantly lower $\pi$ plasmon energy than that found for magnetically aligned SWNT bundles. ${ }^{22}$ These findings support the TEM observations indicating a prevalence of small SWNT bundles and suggest that the bundles are sufficiently small that the overall optical properties of the film are dominated by individual SWNT properties rather than behaving as bulk carbon material. These experimental findings are very interesting for future studies on the 1D properties of SWNTs and promising with regard to applications that take advantage of the unique electronic/optical properties of aligned SWNTs.
Acknowledgment. We thank H. Tsunakawa at the University of Tokyo for assistance with some of the TEM observations.

\section{References and Notes}

(1) Saito, R.; Dresselhaus, G.; Dresselhaus, M. S. Physical Properties of Carbon Nanotubes; Imperial College Press: London, 1998.

(2) Carbon Nanotubes: Synthesis, Structure, Properties, and Applications; Dresselhaus, M. S., Dresselhaus, G., Avouris, Ph., Eds.; SpringerVerlag: Berlin, 2001.

(3) Hwang, J.; Gommans, H. H.; Ugawa, A.; Tashiro, H.; Haggenmueller, R.; Winey, K. I.; Fischer, J. E.; Tanner, D. B.; Rinzler, A. G. Phys. Rev. B 2000, 62, R13310.

(4) Murakami, Y.; Einarsson, E.; Edamura, T.; Maruyama, S. Phys. Rev. Lett. 2005, 94, 087402.

(5) Islam, M. F.; Milkie, D. E.; Kane, C. L.; Yodh, A. G.; Kikkawa, J. M. Phys. Rev. Lett. 2004, 93, 037404.

(6) Murakami, Y.; Chiashi, S.; Miyauchi, Y.; Hu, M.; Ogura, M.; Okubo, T.; Maruyama, S. Chem. Phys. Lett. 2004, 385, 298.

(7) Maruyama, S.; Kojima, R.; Miyauchi, Y.; Chiashi, S.; Kohno, M. Chem. Phys. Lett. 2002, 360, 229.

(8) Hata, K.; Futaba, D. N.; Mizuno, K.; Namai, T.; Yumura, M.; Iijima, S. Science 2004, 306, 1362.

(9) Noda, S.; Hasegawa, K.; Sugime, H.; Kakehi, K.; Zhang, Z.; Maruyama, S.; Yamaguchi, Y. Jpn. J. Appl. Phys. 2007, 46, L399.

(10) Zhang, G.; Mann, D.; Zhang, L.; Javey, A.; Li, Y.; Yenilmez, E.; Wang, Q.; McVittie, J. P.; Nishi, Y.; Gibbons, J.; Dai, H. Proc. Natl. Acad. Sci. 2005, 102, 16141.

(11) Zhong, G.; Iwasaki, T.; Honda, K.; Furukawa, Y.; Ohdomari, I.; Kawarada, H. Jpn. J. Appl. Phys. 2005, 44, 1558.

(12) Eres, G.; Kinkhabwala, A. A.; Cui, H.; Geohegan, D. B.; Puretzky, A. A.; Lowndes, D. H. J. Phys. Chem. B 2005, 109, 16684

(13) Xu, Y.-Q.; Flor, E.; Kim, M. J.; Hamadani, B.; Schmidt, H.; Smalley, R. E.; Hauge, R. H. J. Am. Chem. Soc., 2006, 128, 6560.

(14) Noda, S.; Sugime, H.; Osawa, T.; Tsuji, Y.; Chiashi, S.; Murakami, Y.; Maruyama, S. Carbon 2006, 44, 1414.

(15) Zhang, L.; Tan, Y.; Resasco, D. E. Chem. Phys. Lett. 2006, 422, 198.

(16) Maruyama, S.; Einarsson, E.; Murakami, Y.; Edamura, T. Chem. Phys. Lett. 2005, 403, 320.

(17) Murakami, Y.; Miyauchi, Y.; Chiashi, S.; Maruyama, S. Chem. Phys. Lett. 2003, 377, 49.

(18) Dresselhaus, M. S.; Eklund, P. Adv. Phys. 2000, 49, 705.

(19) Murakami, Y.; Chiashi, S.; Einarsson, E.; Maruyama, S. Phys. Rev. B 2005, 71, 085403.

(20) Murakami, Y.; Maruyama, S. Chem. Phys. Lett. 2006, 422, 575.

(21) Fink, J. Adv. Electron Phys. 1989, 75, 121.

(22) Liu, X.; Pichler, T.; Knupfer, M.; Golden, M. S.; Fink, J.; Walters, D. A.; Casavant, M. J.; Schmidt, J.; Smalley, R. E. Synth. Met. 2001, 121 , 1183.

(23) Kramberger, C.; Shiozawa, H.; Rauf, H.; Grüneis, A.; Pichler, T.; Knupfer, M.; Fink, J.; Büchner, B.; Einarsson, E.; Maruyama, S. Proceedings of the International Winterschool on: "Electronic Properties of Novel Materials: Molecular Nanostructures"; IWEPNM 2007, Kirchberg/Tirol, Austria.

(24) Shyu, F. L.; Lin, M. F. Phys. Rev. B 2000, 62, 8508.

(25) Pichler, T.; Knupfer, M.; Golden, M. S.; Fink, J.; Rinzler, A.; Smalley, R. E. Phys. Rev. Lett. 1998, 80, 4729.

(26) Knupfer, M.; Pichler, T.; Golden, M. S.; Fink, J.; Rinzler, A.; Smalley, R. E. Carbon 1999, 37, 733.

(27) Lin, M. F.; Chuu, D. S. Phys. Rev. B 1998, 57, 10183. 